\title{
Transcription elongation factor P-TEFb is required for HIV-1 Tat transactivation in vitro
}

\author{
Yuerong Zhu, Tsafrira Pe'ery, ${ }^{1}$ Junmin Peng Yegnanarayana Ramanathan, ${ }^{1}$ Nick Marshall, \\ Tricia Marshall, Brad Amendt, Michael B. Mathews, ${ }^{1}$ and David H. Price ${ }^{2}$
}

Department of Biochemistry, University of Iowa, lowa City, lowa 52242 USA; ${ }^{1}$ Department of Biochemistry and M olecular Biology, University of M edicine and Dentistry of N ew Jersey (UMDNJ), N ewark, N ew Jersey 07103 USA

\begin{abstract}
P-TEFb is a key regulator of the process control ling the processivity of RNA polymerase II and possesses a kinase activity that can phosphorylate the carboxy-terminal domain of the largest subunit of RNA polymerase II. Here we report the cloning of the small subunit of D rosophila P-TEFb and the finding that it encodes a Cdc2-related protein kinase. Sequence comparison suggests that a protein with $72 \%$ identity, PITALRE, could be the human homolog of the D rosophila protein. Functional homology was suggested by transcriptional analysis of an RNA polymerase II promoter with HeLa nuclear extract depleted of PITALRE. Because the depleted extract lost the ability to produce long DRB-sensitive transcripts and this loss was reversed by the addition of purified D rosophila P-TEFb, we propose that PITALRE is a component of human P-TEFb. In addition, we found that PITALRE associated with the activation domain of HIV-1 Tat, indicating that P-TEFb is a Tat-associated kinase (TAK). An in vitro transcription assay demonstrates that the effect of Tat on transcription elongetion requires P-TEFb and suggests that the enhancement of transcriptional processivity by Tat is attributable to enhanced function of P-TEFb on the HIV-1 LTR.
\end{abstract}

[Key Words: P-TEFb; transcription elongation factor; RN A polymerase II; HIV-1 Tat; transactivation]

Received July 22, 1997; revised version accepted August 21, 1997.

The intricate pattern of gene expression exhibited by all eukaryotic organisms is accomplished, in part, through control of the steps required to generate mature mRN As. It is increasingly clear that one of the major regulatory processes affects the elongation potential of RNA polymerase II after initiation (Spencer and Groudine 1990; Kerppola and Kane 1991; Wright 1993; Bentley 1995). N egative factors instigate this el ongation control process by limiting the processivity of RN A polymerase II, leading to abortive elongation, a process characterized by premature termination (Marshall and Price 1992). One such factor, Drosophila factor 2, causes transcri pt release (Xie and Price 1996). Positive factors, exemplified by Drosophila positive transcriptional elongation factor b (P-TEFb; Marshall and Price 1995), trigger the escape into productive elongation and allow the generation of transcripts that extend beyond the $3^{\prime}$ ends of mature mRN As. Pol ymerases that have made the transition into productive elongation are further affected by elongation factors, such as S-II (Guo and Price 1993), TFIIF (Kephart et al. 1994), ELL (Shilatifard et al. 1996), and elongin (Aso et al. 1995); these factors increase the efficiency of transcription elongation (Reines et al. 1996). Whereas the

${ }^{2}$ Corresponding author.

E-MAIL david-price@uiowa.edu; FAX (319) 335-9570. effects of the latter factors are important, the transition from abortive to productive el ongation is the major regulated step (Wright 1993).

The carboxy-terminal domain (CTD) of the largest subunit of RN A polymerase II plays an important role in the elongation control process. It was first noticed that phosphorylation of the CTD occurred at the same time as the transition into productive elongation (O'Brien et al. 1994; Dahmus 1996). It was then found that an intact CTD was required for the elongation control process (Chun and Jeang 1996; Marshall et al. 1996). A connection between elongation control and CTD phosphorylation came with the discovery that Drosophila P-TEFb had CTD kinase activity ( $M$ arshall and Price 1995; $M$ arshall et al. 1996). The CTD kinase activity and transcriptional function of P-TEFb are blocked by low levels of 5,6-dichloro-1- $\beta$-D-ribofuranosylbenzimidazole (DRB), a nucleoside analog that inhibits CTD phosphorylation (Egyhazi et al. 1996) and the production of mRNAs (Tamm and Kikuchi 1979) in vivo.

HIV-1 Tat greatly stimulates gene expression from the viral promoter located in the long terminal repeat (LTR) of the human immunodeficiency virus (HIV) genome (Jones and Peterlin 1994). The primary effect of Tat is to increase the processivity of RNA polymerases that otherwise would prematurely terminate after the synthesis 
of short nascent transcripts (Kao et al. 1987; Laspia et al . 1989; Feinberg et al. 1991; Marciniak and Sharp 1991; Laspia et al. 1993). The elongation effect requires an RNA element, transactivation response (TAR), that forms a stem and loop structure with which Tat can associate (Jones and Peterlin 1994). Consistent with its effect on elongation $T$ at has been found to be an integral component of activated el ongation complexes (Keen et al. 1996). The function of T at in el ongation is similar to the function of P-TEFb in that the transcriptional stimulation by Tat is sensitive to DRB (Braddock et al. 1991; Marciniak and Sharp 1991) and requires the CTD of the largest subunit of RNA polymerase II (Chun and Jeang 1996; Parada and Roeder 1996; Y ang et al . 1996). In vitro (Herrmann and Rice 1993, 1995; Chun and Jeang 1996) and in vivo (Y ang et al. 1996) Tat specifically associates with a serine/ threonine kinase through its activation domain. Like P-TEFb, this Tat-associated kinase (TAK) is sensitive to DRB (Y ang et al . 1996). It has been suggested that $T$ at might function by recruiting TAK to phosphorylate the CTD (Y ang et al . 1996). T at has also been shown to affect initiation (Laspia et al. 1989; Veschambre et al. 1995). Tat can associate with transcription preinitiation complexes and this association does not requi re the TAR element (Garcia-Martinez et al. 1997a). Consistent with a function in initiation, recent results have shown that Tat can interact with the RNA polymerase II holoenzyme in the absence of TAR (Cujec et al. 1997).

Besides the involvement of P-TEFb in el ongation control (Marshall and Price 1995; Marshall et al. 1996), TFIIH has al so been implicated in the process. There is a correlation between activators that stimulate el ongation and their ability to associate with TFIIH (Blau et al. 1996; T suchiya et al. 1996). In addition, anti bodies against subunits of TFIIH inhibited elongation of transcription of the c-myc gene when both were injected into Xenopus oocytes (Yankulov et al. 1996). Parada and Roeder (1996) and Garcia-Martinez et al. (1997b), but not Yang et al. (1996), found that TFIIH could associate with Tat and was required for Tat-stimulated transcription. Strong evidence links TFIIH with a late event in initiation, promoter escape (Goodrich and Tjian 1994), whereas P-TEFb has been shown to function during elongation ( $M$ arshall and Price 1995). It is possible that both factors are involved, and they act independently or one may regulate the activity of the other.

In this paper we report the cloning of the kinase subunit of Drosophila P-TEFb and show that a previously known protein, PITALRE, is the homologous human PTEFb catalytic subunit. We provide several lines of evidence indicating that P-TEFb associates with the HIV-1 Tat. Furthermore, we show that human P-TEFb is required for Tat-mediated stimulation of transcription elongation. Our results suggest that the ability of Tat to increase the processivity of RNA polymerase II is mediated by its ability to enhance the function of the elongation control factor, P-TEFb.

\section{Results}

Cloning of the kinase subunit of Drosophila P-TEFb

To further our understanding of the function of P-TEFb we cloned a cDNA encoding the small subunit of the Drosophila factor. The two subunits of purified P-TEFb were separated by gel el ectrophoresis, and the small subunit was excised and subjected to protein sequencing. The peptide sequence information was used in the cloning of full-length CDNA, as described in Materials and $M$ ethods. The deduced ami no acid sequence (Fig. 1) identified the small subunit of Drosophila P-TEFb as a member of the $\mathrm{Cdc}$-like cyclin dependent kinase family with $>40 \%$ identity to Schizosaccharomyces pombe Cdc2. A search of the protein database reveal ed a human protein, PITALRE (Grana et al . 1994), that exhibits $72 \%$ identity and $83 \%$ similarity to the Drosophila protein (Fig. 1). The high level of sequence similarity indicated that PITALRE is a potential homolog of the small subunit of

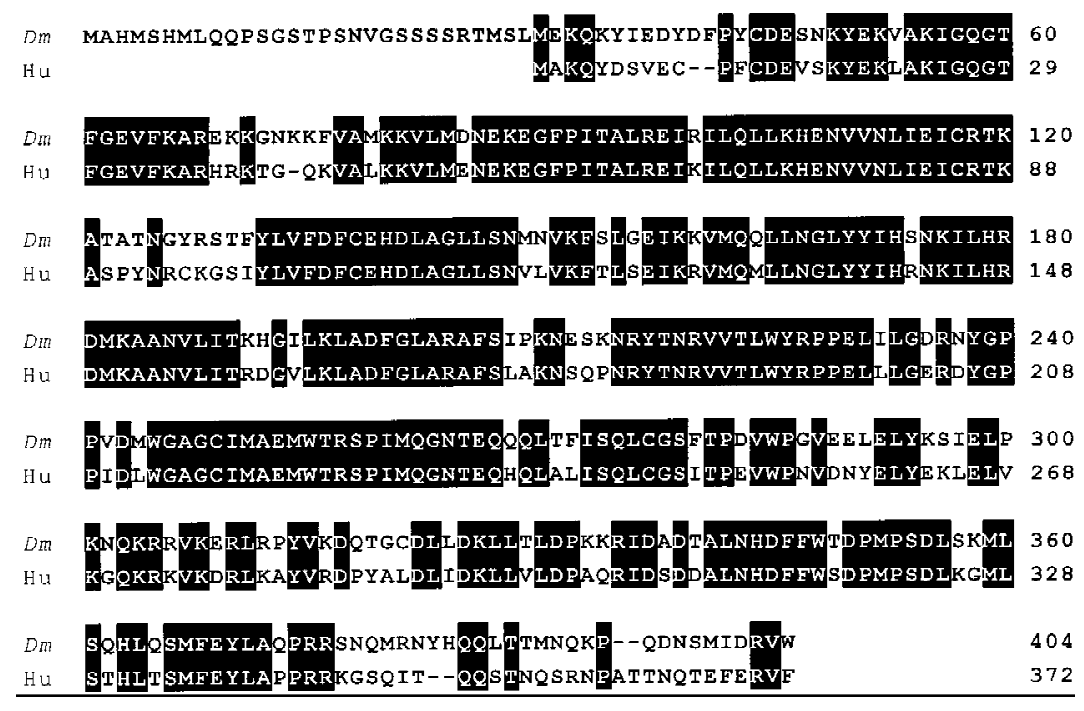

Figure 1. The small subunit of Drosophila P-TEFb is similar to PITALRE. (Dm) Small subunit of Drosophila P-TEFb; $(\mathrm{Hu})$ human PITALRE. Black reverse shading indicates identity. 
Drosophila P-TEFb and therefore may be a component of human P-TEFb. Two kinases from Saccharomyces cerevisiae, SGV1 (Irie et al. 1991) and CTK1 (Sterner et al. 1995), each share $43 \%$ identity with PITALRE and the small subunit of Drosophila P-TEFb. Although sequence similarity does not allow the prediction of a potential yeast homolog, CTK1 has recently been demonstrated to increase the el ongation efficiency of RN A polymerase II (Lee and Greenleaf 1997).

\section{PITALRE is a component of human P-TEFb}

Drosophila P-TEFb functions during elongation by increasing the processivity of RN A polymerase II, allowing the synthesis of long transcripts ( $M$ arshall and Price 1995; $M$ arshall et al. 1996). P-TEFb function is indicated by the generation of DRB-sensitive runoff transcripts during transcription in vitro. If PITALRE is the functional homolog of the small subunit of Drosophila P-TEFb, removal of PITALRE from HeLa nuclear extract (HNE) should eliminate DRB-sensitive runoff transcripts. To test this we immunodepleted PITALRE from HNE with antibodies directed against the last 20 amino acids of PITALRE that are not shared with other known kinases (Fig. 2A). Western blot analysis indicated that PITALRE was removed by anti-PITALRE antibodies to levels below detection but not by control antibodies (Fig. 2B). The depleted HN E was unable to generate DRB-sensitive 633-nucleotide runoff transcripts from an HIV-1 LTR template in a pulse/chase transcription reaction (Fig. 2C). Addition of pure Drosophila P-TEFb to the de-

A
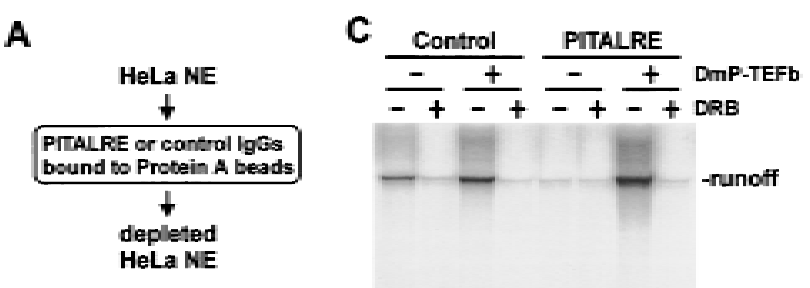

$\mathbf{B}$
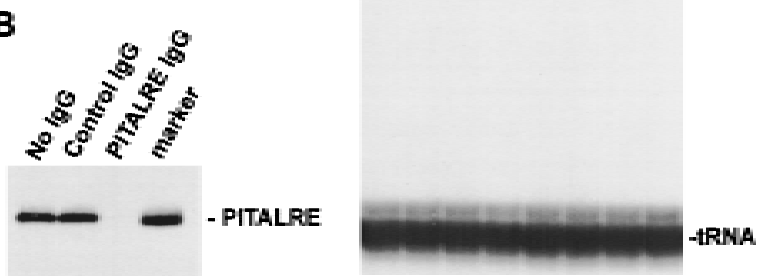

Figure 2. Human P-TEFb is required for the generation of DRBsensitive runoff transcripts. (A) Immunodepletion. HNE was passed over two successive protein A columns containing either affinity-purified PITALRE-CT IgG or affinity-purified rabbit anti-goat IgG (control) as diagramed. (B) Western blot of HNE depleted with indicated antibodies probed with PITALRE-CT antibodies. (M arker) Bacterially expressed PITALRE. (C) Transcriptional activity of human P-TEFb. The pulse-chase protocol is described in M aterials and M ethods. (DmP-TEFb) Drosophila P-TEFb; (tRN A) tRN A labeled with [ $\left.{ }^{32} \mathrm{P}\right]$ CTP during transcription reaction (recovery control). pl eted extract restored DRB-sensitive transcription (Fig. 2C). These results indicate that depletion of PITALRE abolished human P-TEFb activity, supporting the hypothesis that PITALRE is a component of human PTEFb.

PITALRE was not known previously to be a CTD kinase. Therefore, we examined the material immunoprecipitated during the depletion of HNE for CTD kinase activity. The antibody-loaded beads containing PITALRE were washed extensively with high salt and subjected to a CTD kinase assay. Similar to Drosophila P-TEFb, beads containing PITALRE (together with any other strongly associated proteins) were able to convert the largest subunit of Drosophila RNA polymerase II to the hyperphosphorylated IIo form (Fig. 3A). Control beads were inactive. As expected, all phosphorylation was sensitive to $50 \mu \mathrm{M}$ DRB. In the control reaction with Drosophila P-TEFb autophosphorylation of both subunits (43 and $124 \mathrm{kD}$ ) was seen. In the reaction with beads containing PITALRE antibodies, the 40-kD PITALRE, a band of similar size to the large subunit of Drosophila P-TEFb, and several other bands were phosphorylated.

To examine the association of other proteins with PITALRE, immunoprecipitates were washed with buffer containing noni onic detergents and increasing amounts of salt. The proteins associated with the beads were analyzed by SDS-PAGE, followed by silver staining (Fig. 3B). When no salt was present many proteins were retained on the beads. Most of these proteins were removed by washing with $200 \mathrm{~mm} \mathrm{~N} \mathrm{aCl}$. N o changes occurred in the proteins visible after a wash with buffer containing $\mathrm{N} \mathrm{aCl}$ higher than $400 \mathrm{~mm}$. Besides immunogl obulin heavy and light chain and PITALRE (40 kD), proteins with sizes 87, 105,133 , and $140 \mathrm{kD}$ were found. When rabbit anti-goat IgG control beads were used no other proteins except for the immunoglobulins were seen after high salt washes (data not shown). The immunoprecipitates were incubated with $\left[\gamma^{-32} \mathrm{P}\right]$ ATP to determine which proteins became phosphorylated (Fig. 3C). The beads washed without salt carried out extensive phosphorylation of many protein substrates with little DRB sensitivity. After washing with $400 \mathrm{~mm}$ or higher concentration of $\mathrm{NaCl}$, only a few proteins were labeled and all phosphorylation was DRB sensitive. Of the major proteins associated with PITALRE, only the $105-k D$ protein was not phosphorylated. At all salt concentrations the partially DRBsensitive phosphorylation of a 207-kD protein was observed. Except for PITALRE the identity of the other proteins is unknown. The sizes of these proteins do not correlate with subunits of other known basal transcription factors. The other proteins could be constituents of a larger complex containing P-TEFb or different complexes containing PITALRE.

A distinguishing characteristic of P-TEFb is its sensitivity to the kinase inhibitors $D R B$ and $\mathrm{H}-8$. In vitro transcription and the CTD kinase activity of Drosophila $\mathrm{P}-\mathrm{TEFb}$ are both inhibited by these two compounds, and in both assays DRB is 10-fold more potent than $\mathrm{H}-8$ (Marshall and Price 1995; M arshall et al. 1996). The ef- 
A



B

Silver Stain $\mathrm{mWNaCi}$ wash



C

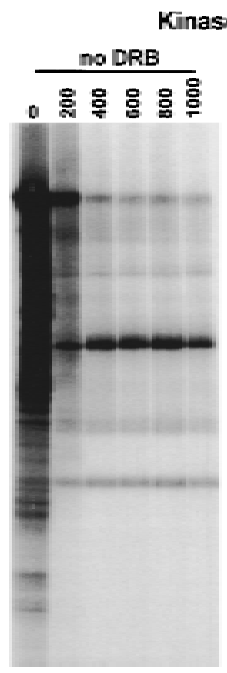

Figure 3. Immunoprecipitation of human P-TEFb. (A) CTD kinase assay. Purified Drosophila RNA polymerase II (Price et al. 1987; M arshall and Price 1995) was used as substrate. (DmP-TEFb) Drosophila P-TEFb; (Control) beads after depletion of HNE by affinity-purified rabbit anti-goat IgG; (HuPTEFb) beads after depletion of HNE by PITALRE-CT IgG. Products were analyzed by $6 \%-15 \%$ gradient SDS-PAGE. Selected size standards from the 10-kD ladder are indicated and apply to all gels. (B) Silverstained SDS-PAGE of proteins bound to PITALRE-CT IgG beads after washing with a buffer containing $20 \mathrm{~mm}$ HEPES (pH 7.6), $0.5 \%$ N P-40, $1 \%$ Triton X-100, and $5 \mathrm{~mm}$ DTT and the indicated amount of $\mathrm{NaCl}$. Sizes of proteins remaining after high salt wash are indicated. Bands marked with an asterisk $(*)$ are from IgG. (C) Kinase assay with only endogenous substrates of the fractions analyzed in $\mathrm{B}$ either without or with $50 \mu \mathrm{M}$ DRB, as indicated. fect of DRB and $\mathrm{H}-8$ were determined on transcription in the HNE (Fig. 4A,B) and on kinase assays using the immunoprecipitated human P-TEFb (Figure 4C,D). In both assays DRB was the more effective inhibitor. DRB and H8 compete with ATP for binding to the kinase active site; therefore, it is inappropriate to compare directly the $50 \%$ inhibition points under different conditions, especially if different concentrations of ATP are used (Marshall et al. 1996). However, the ratio of $50 \%$ points for different compounds under identical conditions can be compared. This ratio is more likely to be independent of assay condition. The ratio of $50 \%$ inhibition points $(\mathrm{H}$ 8/DRB) was $23 \mu \mathrm{m} / 1 \mu \mathrm{m}=23$ for the transcription assay and $16 \mu \mathrm{m} / 0.65 \mu \mathrm{m}=25$ for the CTD kinase assay. These ratios are similar to each other, suggesting that the same kinase, namely human P-TEFb, is being inhibited in both assays. Consi dering the sequence similarity between the small subunit of Drosophila P-TEFb and PITALRE, the functional similarity between Drosophila P-TEFb and the activity removed from HNE by PITALRE antibodies, and the presence of other potential subunits in the immunoprecipitates, we conclude that PITALRE is a component of human P-TEFb.

P-TEFb specifically associates with the activation domain of HIV Tat

Several lines of evidence led us to hypothesize that PTEFb might associate with the viral transactivator Tat. Tat transactivation is sensitive to DRB (Braddock et al. 1991; Marciniak and Sharp 1991) and requires the CTD (Chun and Jeang 1996; Parada and Roeder 1996; Yang et al. 1996), and Tat associates with a DRB-sensitive CTD kinase (TAK) (Herrmann and Rice 1993, 1995; Chun and

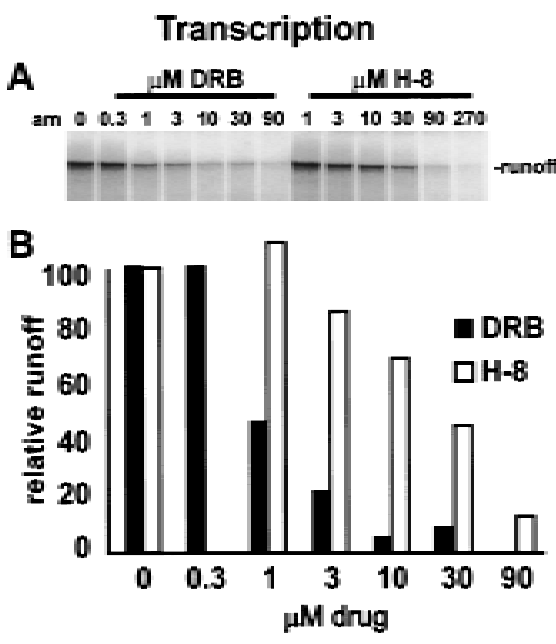

Figure 4. DRB and $\mathrm{H}-8$ inhibition of transcription and human P-TEFb activity. (A,B) Transcription of HIV LTR template (633nucleotide runoff); (C,D) CTD kinase assay using immunoprecipitated human P-TEFb and Drosophila RN A polymerase II as substrate, as in Fig. 3A. (B,D) Plot of radioactivity in runoff or polymerase llo after quantitation using a Packard Instantlmager and normalization to the starting amount (100). 


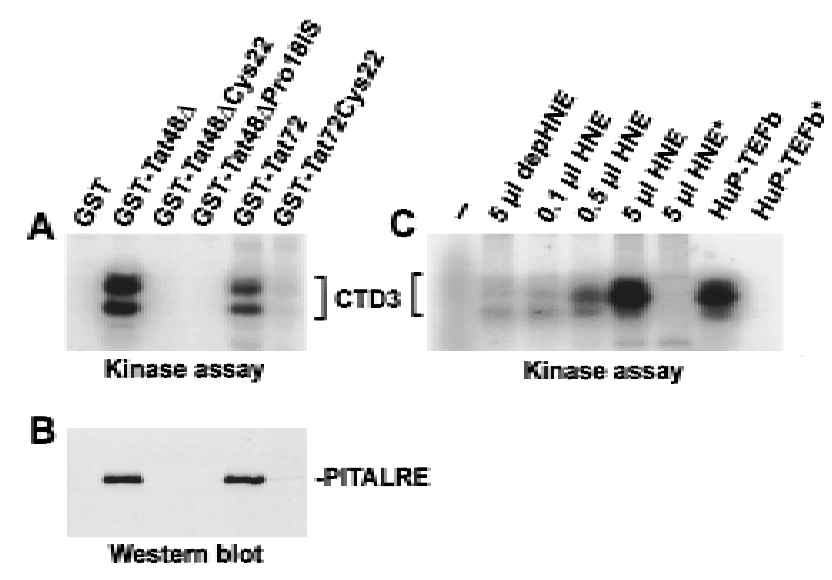

Figure 5. PITALRE associates with the activation domain of HIV-1 Tat. (A) TAK activity assay using CTD3 peptide as substrate. The indicated GST Tat or Tat mutant proteins were used. Both Tat72 and Tat $48 \Delta$ have intact transactivation domains, whereas other constructs have mutations that abolish transactivation. (Tat72) HIV-1 Tat containing residues 1-72; (Tat48D) the activation domain of HIV-1 Tat containing residues 1-48; (Cys22) mutant Tat containing Gly instead of Cys at position 22; (Prol8IS) Tat mutant containing Glu and Phe insertion after Pro-18. (B) Western bl ot of the same samples using antibodies against PITALRE-CT. (C) TAK activity assays using GST-T at $48 \Delta$ were performed with whole or depleted (depHNE) HNE. In the kinase assay CTD3 peptide was used as substrate. $(\rightarrow$ N o kinase added; (*) no CTD3 peptide added. Human P-TEFb (HuP-TEFb) was immunoprecipitated from the equivalent of 1 $\mu$ of HNE.

Jeang 1996; Y ang et al. 1996). The hypothesis was tested by ascertaining whether the human P-TEFb kinase associates with Tat during incubation with HeLa extracts. Glutathione beads containing various glutathione Stransferase (GST)-Tat fusion proteins were incubated with HeLa extract and washed extensively. Proteins associated with Tat constructs containing an intact activation domain (Tat72 and Tat48 $\Delta$ ) were able to phosphorylate the synthetic peptide CTD3 (Fig. 5A), as well as RN A polymerasell (data not shown). GST-T at fusions containing mutations in the activation domain that abolish T at transactivation (Rice and Carl otti 1990; Herrmann and Rice 1995) were not able to pull down TAK (Fig. 5A). The proteins associated with the Tat constructs were probed with anti-PITALRE antibody by Western blot analysis (Fig. 5B). PITALRE was detected only when the constructs contained an intact Tat transactivation domain. This suggests that P-TEFb is a TAK.

When human P-TEFb was depleted from HNE by antibodies to PITALRE, TAK activity (assayed by GSTTat $48 \Delta$ pull-down) was reduced to $<\%$ of that found in the intact extract (Fig. 5C, cf. the lane using $5 \mu \mathrm{l}$ of depleted extract and that using $0.1 \mu \mathrm{l}$ of whole extract). This strongly suggests that under the conditions used human P-TEFb is the predominant CTD kinase that associates with Tat. Others have shown that TFIIH can associate with Tat (Parada and Roeder 1996; Garcia-M artinez et al . 1997b), but under the conditions used, we did not detect any p62 subunit of TFIIH bound to Tat nor did we detect a reduction in the amount of the subunit in the PITALRE-depl eted extract. In addition, when glutathione beads containing TAK were probed with antibodies to all three subunits of CAK (CDK7, cyclin H, Mat1; supplied by D. Morgan, University of California, San Diego) we found no evidence of the TFIIH-associated kinase (data not shown).

To further confirm that human P-TEFb can associate with Tat, TAK was partially purified from $\mathrm{HeLa}$ cells by sequential chromatography on DEAE, heparin, and SP resins. Fractions eluted from the second and third columns, Heparin and SP were assayed for TAK activity and probed for human P-TEFb (Fig. 6). In the eluate of both columns, TAK activity assayed by GST-Tat $48 \Delta$ pulldown (Fig. 6A,D) correlated with P-TEFb determined by Western analysis using two different preparations of PITALRE antibodies (Fig. 6B,C,E,F). A GST construct with a mutation in the Tat activation domain did not become associated with CTD kinase activity when incubated with the same column fractions (data not shown). In addition, we compared the drug sensitivity of TAK and human P-TEFb. As expected, TAK activity was inhibited by DRB and $\mathrm{H}-8$ in a manner similar to $\mathrm{P}-\mathrm{TEFb}$ (data not shown).

Human P-TEFb is required for Tat-stimulated elongation

To investigate the function of P-TEFb in Tat transactivation directly, we compared the effect of Tat on initiation and elongation in whole or PITALRE-depleted extracts (Fig. 7). T wo different DN A templates were used. TheTAR template contai ned HIV-1 LTR sequences from -475 to +76 , whereas the $\Delta T A R$ template lacked the se quence encoding TAR and contained HIV-1 LTR se quences from -475 to +19 . With HNE (not depleted) and a continuous labeling protocol (Fig. 7A), $10 \mathrm{ng} / \mu \mathrm{l}$ of Tat stimulated the generation of runoff $\sim 12$-fold (Fig. 7C,

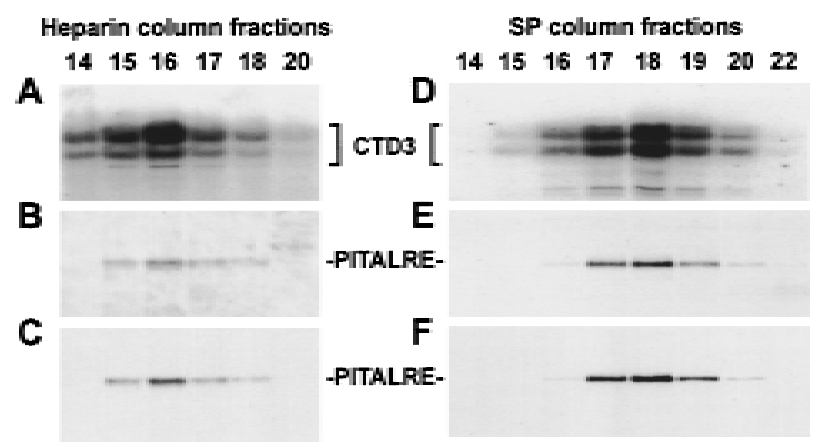

Figure 6. PITALRE coel utes with TAK activity. (A-C) Heparin column fractions; (D-F) SP column fractions. TAK activity was assayed with GST-Tat48D across both columns using CTD 3 as the kinase substrate $(A, D)$; PITALRE was detected in Western blots using antibodies against whole PITALRE $(B, E)$ or PITALRE-CT, (C,F). 
A



B

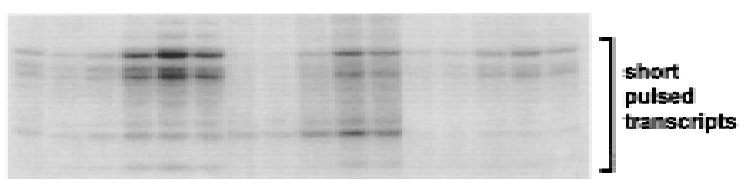

C

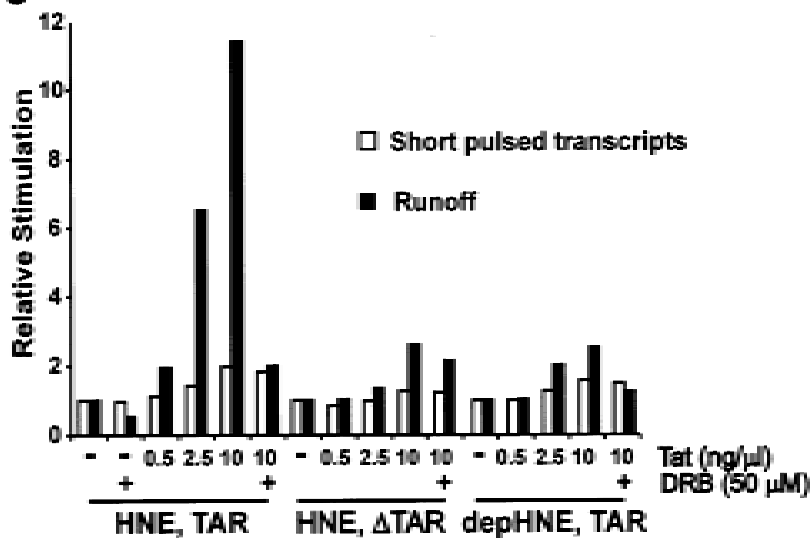

Figure 7. P-TEFb is required for Tat transactivation in vitro. (A) In vitro Tat transactivation using a continuous labeling protocol with the indicated templates and extracts. Runoff transcripts were analyzed in a $6 \%$ TBE/ urea gel. (B) Similar reaction mixtures as in A were subjected to a 2-min pulse, and the short transcripts generated ( $<30$ nucl eoti des) were analyzed in an $18 \%$ TBE/ urea gel. (C) Quantitation data for A and B. The amount of runoff or short transcripts for each template/extract combination was normalized to the corresponding lane with no DRB or Tat added.

solid bars). This effect of Tat was mostly inhibited by 50 $\mu \mathrm{M} D R B$ and required the TAR sequence. The DRB sensitivity of Tat-stimulated runoff was similar to that determined for the low level of runoff from the HIV LTR in the absence of Tat shown in Figure 4 (data not shown). When PITALRE-depleted extract was used, the majority of the stimulatory effect of Tat was abolished. This result strongly suggests that human P-TEFb is requi red for efficient transactivation by $T$ at. A dd-back of D rosophila $\mathrm{P}$-TEFb to the depleted extract stimulated runoff in the presence or absence of Tat, as seen in Figure 2, but did not restore the ability of Tat to specifically enhance elongation (data not shown). This suggests that other required factors were removed by the depletion of PITALRE or that Drosophila P-TEFb lacks appropriate domains required for interaction with Tat or other cofactors. Addition of the high salt-washed PITALRE immunoprecipitate had no effect on runoff in the presence or absence of Tat (data not shown), indicating that immobilization had a negative impact on the function of P-TEFb. Although consistent with an effect on elongation, the experiment shown in Figure 7A did not rule out the possibility that the major effect of Tat was on initiation.

From the autoradiograph (Figure 7A) and the normalized quantitation data graphed in Figure 7C (solid bar) it was obvious that Tat had a modest effect on transcription (about twofold increase of runoff) in the presence of $50 \mu \mathrm{M} D R B$, in the presence of $\triangle T A R$ template, or even in the absence of human P-TEFb. To further analyze the effect of Tat on transcription, we performed a similar set of experiments, as shown in Figure 7A, except that a pulse-only transcription protocol was used instead of a continuous labeling protocol (Fig. 7B). Quantitation of the short pulsed transcripts ( $<20$ nucleotides in length) gives the efficiency of initiation. Under these conditions it was clear that increasing Tat had the effect of increasing transcription initiation (Fig. 7B and open bars in C). At the highest level of Tat used $(10 \mathrm{ng} / \mu \mathrm{l})$ initiation increased about twofold compared to initiation when no $T$ at was added. This effect on initiation did not require TAR (Fig. 7B,C). M oreover, the effect of Tat on initiation was not sensitive to DRB and did not require P-TEFb. The effect of Tat on initiation can be used to explain the slight effect of $T$ at on the generation of runoff in the presence of DRB, $\triangle T$ TAR template, or depleted HNE (Fig. 7A). The two assays taken together indicate that the major effect of $\mathrm{T}$ at is on el ongation, although initiation is slightly affected. Most importantly, P-TEFb is required for the effect of $T$ at on el ongation but not for its effect on initiation.

\section{Discussion}

We have cloned the small subunit of Drosophila P-TEFb and identified it as the counterpart of two human protein kinases whose mutual identity was not recognized previously. Sequence similarity suggested that the small subunit of Drosophila P-TEFb was the homolog of PITALRE, a Cdc2-related kinase of hitherto unknown function. This was confirmed when Drosophila P-TEFb was able to functionally replace the transcriptional activity removed from HeLa nucl ear extract with PITALRE antibodies. Using immunological and biochemical tests we found that human P-TEFb was a TAK. Furthermore, we showed that the effect of Tat on transcription elongation was dependent on P-TEFb. These results provide a foundation for understanding the mechanism of action of Tat in HIV transcriptional transactivation and should facilitate the development of defined in vitro Tat transactivation systems and of drugs aimed at blocking Tat transactivation.

All three of these activities, P-TEFb, PITALRE, and TAK, previously were known to be associated with a 42to 43-kD phosphoprotein that is now characterized as the kinase subunit of the human P-TEFb complex. In Drosophila this complex contains two subunits of 43 and 124 kD (Marshall and Price 1995), whereas the human P-TEFb complex is less well defined. The small subunit is found in association with several high molecular weight polypeptides (Fig. 3; Grana et al. 1994; Garriga et al. 1996a). The roles of the additional polypeptides are 
unknown, but it is possible that they interact with other components of the transcriptional machinery and/or regulate kinase activity. Because PITALRE has similarity to other cyclin-dependent kinases it is likely that one of these associated proteins is a cyclin subunit. PITALRE with its associated proteins is a proline-directed serine/ threonine kinase (Garriga et al . 1996b) that, like its Drosophila counterpart, is able to hyperphosphorylate the CTD of RN A polymerase II (Figs. 3 and 5; Herrmann and Rice 1995; M arshall et al. 1996). Although proteins such as the retinoblastoma protein pRB (but not histone $\mathrm{H} 1$ ) can serve as substrates (Grana et al. 1994), at least in vitro, the link between transcription elongation and RNA polymerase II phosphorylation makes it likely that the CTD is a major substrate for the P-TEFb kinase in vivo.

CTD hyperphosphorylation is associated with processive elongation (Dahmus 1996). Correspondingly, the CTD is essential for P-TEFb-mediated transition to productive el ongation in vitro ( $M$ arshall et al. 1996) and for Tat transactivation in vivo and in vitro (Parada and Roeder 1996; Y ang et al. 1996). Drosophila P-TEFb acts at an early stage of elongation to allow transcription complexes to make the transition to productive elongation (Marshall et al. 1996), and results presented here extend this finding to the human transcription system (Fig. 2). Similarly, Tat binds to short nascent transcripts (Jones and Peterlin 1994) and overcomes promoter-proximal termination (Kao et al. 1987; Laspia et al. 1989, 1990). A priori, the properties of human P-TEFb make it an attractive candi date for the critical agent recruited to the elongating complex by Tat. However, Tat has been reported to associate with numerous components of the transcription apparatus, as well as other proteins (Jones and Peterlin 1994).

Several lines of evidence indicate that P-TEFb is involved in the stimulation of processivity brought about by Tat. P-TEFb binds specifically to the activation domain of Tat, and the interaction is el iminated by mutations that abrogate transactivation (Fig. 5; Herrmann and Rice 1993). The interaction of TAK with Tat survives exhaustive washing under stringent conditions and has been observed in vitro (Herrmann and Rice 1993, 1995; Chun and Jeang 1996) and in vivo (Yang et al. 1996). Furthermore, TAK (P-TEFb) binds to the Tat proteins of HIV-2 and equine infectious anemia virus (Herrmann and Rice 1993, 1995), which both appear to function through the same cofactor as HIV-1 Tat (Carroll et al. 1992; M adore and Cullen 1993). A second line of support derives from experiments with the nucleoside analog DRB. The enhanced processivity brought about by PTEFb and by Tat are both selectively sensitive to low levels of DRB (Figs. 4 and 7; Braddock et al. 1991; Marciniak and Sharp 1991; M arshall and Price 1992). Correspondingly, the CTD kinase activities of Drosophila PTEFb and TAK are sensitive to low levels of DRB and related inhibitors (Fig. 4; Herrmann and Rice 1995; Egyhazi et al. 1996; Marshall et al. 1996). Perhaps the most compelling argument for the rol e of P-TEFb in T at transactivation comes from depl etion experiments (Figs. 2, 3,
5, and 7). Antibodies directed against a 20-residue peptide from the carboxyl terminus of PITALRE simultaneously removed TAK activity and rendered the HeLa nuclear extract dependent on P-TEFb for efficient elongation. P-TEFb could be detected in the immunoprecipitates by silver staining and CTD kinase assay. M ost importantly, HNE depleted of P-TEFb was unable to support Tat transactivation (Fig. 7A).

We observed that Tat stimulated transcriptional initiation as well as elongation in our in vitro transcription assay. This has been observed before (Laspia et al. 1989; Veschambre et al. 1995) and is consistent with the fact that Tat interacts with the RN A polymerase II holoenzyme (Cujec et al. 1997) and associates with transcription preinitiation complexes (Garcia-Martinez et al. 1997a). However, Tat stimulated initiation only $\sim 2$-fold, whereas its runoff transcripts increased >10-fold (Fig. 7), consistent with previous results (Kao et al. 1987; Laspia et al . 1989; Feinberg et al. 1991) that the major effect of Tat is on elongation. The fact that TAR and P-TEFb are only required for the effect on elongation further differentiate the effect of T at on initiation and el ongation. The stimulation of initiation by Tat may be mediated through its interaction with SP1 (Jeang et al. 1993), TATA-binding protein (TBP) (Kashanchi et al. 1994; Veschambre et al. 1995), the p62 subunit of TFIIH (Parada and Roeder 1996), or RN A polymerase II (Mavankal et al. 1996). The effect of Tat on el ongation is likely mediated by the interaction of TAR RNA with a complex containing Tat and P-TEFb. The complex may contain other cofactors such as Tat-SF1 (Zhou and Sharp 1996), which is required for Tat transactivation. Supporting this idea, Tat-SF1 has been shown to associate with a kinase that is also required for Tat transactivation (Zhou and Sharp 1996). A model integrating the effects on initiation and elongation is difficult to formulate. However, work of Hernandez and coworkers has indicated that Tat might affect the way RNA polymerase II initiates at the HIV LTR under different conditions because of the presence of a DN A el ement around the start point of transcription that induces short transcripts (IST) (Cullen 1993; Sheldon et al. 1993; Pendergrast and Hernandez 1997). The different types of early el ongation complexes could have different receptivity to P-TEFb action.

Although P-TEFb is required for normal elongation control and Tat transactivation, an important question is whether Tat enhances the function of P-TEFb specifically during transcription of the HIV LTR. The association of P-TEFb with the activation domain of Tat suggests that an enhancement of activity is possible. The results in Figure 7 also strongly suggest that Tat allows P-TEFb to function more effectively. If P-TEFb merely performed its normal function the amount of DRB-sensitive runoff generated would be related to the number of initiation events. Because Tat stimulated initiation about twofold from the LTR either with or without TAR, the effect on DRB-sensitive runoff shoul d have been twofold. However, when the TAR sequence was present Tat increased DRB-sensitive runoff transcripts $\sim 12$-fold. The most likely explanation is that Tat enhanced the func- 
tion of P-TEFb. Because P-TEFb is limiting in nuclear extracts (Marshall and Price 1992, 1995) Tat may allow the available P-TEFb to be recruited to early el ongation complexes containing TAR RNA. Consistent with the idea that the enhancement of P-TEFb function by $T$ at is attributable to increased local concentration of P-TEFb, we have observed a stimulation of DRB-sensitive runoff by increasing the concentration of P-TEFb in the extract in the absence of Tat (see Fig. 2). One caveat to the interpretation of our results is that the method used to measure initiation only looked in a 2-min window. It is possible that the effect of Tat on initiation during the 20-min continuous labeling period used to examine Tat transactivation might have been different. Further experiments will be needed to resolve this and other uncertainties about the mechanism of the enhancement of P-TEFb function.

Although we have shown that P-TEFb plays a role in elongation control, others have implicated TFIIH in the process. In our work with P-TEFb we have not uncovered a specific function for TFIIH in elongation control, although it is present in all reactions because of its initiation requirement. Drosophila P-TEFb has been shown to increase the processivity of RNA polymerase II when added to in vitro reactions after the initiation phase was complete ( $M$ arshall et al. 1996). In the same study purified Drosophila TFIIH was unable to functionally substitute for P-TEFb even though it possessed a similar level of CTD kinase activity (M arshall et al. 1996). In comparing work from a number of laboratories, a point of confusion is the sensitivity of the two kinases to the competitive inhibitors DRB and H8. Although it has been suggested that TFIIH is the DRB-sensitive kinase (Yankulov et al. 1995), we showed in the Drosophila system that P-TEFb was significantly more sensitive to DRB than was TFIIH (M arshall et al. 1996). A comparison of the DRB sensitivity of human TFIIH and P-TEFb kinase activity has not been made directly using identical conditions; however, using a standard CTD kinase assay (10 $\mu$ M ATP) DRB had a $50 \%$ inhibition point of $<1$ $\mu \mathrm{M}$ against $\mathrm{P}-\mathrm{TEFb}$, which is significantly lower than the 10-50 $\mu \mathrm{M}$ reported for TFIIH under similar (7.5 $\mu \mathrm{M}$ ATP) conditions (Yankulov et al . 1995). Therefore, we suggest that caution should be exercised when interpreting experiments utilizing kinase inhibitors such as DRB and H8. A second point of confusion is the association of the CTD kinases with Tat. Neither we (T. Pe'ery, unpubl.) nor Yang et al. (1996) found TFIIH in association with Tat. However, Parada and Roeder (1996) as well as Garcia-M artinez et al. (1997b) reported that T FIIH associated with Tat and was required for Tat-stimulated transcription. Unlike our results in which P-TEFb associated with Tat in the presence of a crude nuclear extract, the latter group found that TFIIH only associated with Tat after partial purification (Garcia-M artinez et al . 1997b). Parada and Roeder (1996) only found hyperphosphorylation of the CTD when a crude fraction of TFIIH containing other factors was used. Despite the confusion, strong evidence for the involvement of TFIIH in elongation control comes from experiments that do not involve kinase in- hibitors but, rather, antibodies against subunits of TFIIH (Yankulov et al. 1996). Yankulov et al. (1996) showed that injection of antibodies against TFIIH had an effect on transcription similar to DRB. Our previous results and those presented here do not rule out a significant role for the kinase activity of TFIIH in elongation control or Tat transactivation, but rather, indicate only that another kinase, P-TEFb, is required. N ow that the kinase subunit of P-TEFb has been identified, examining the potential interplay between TFIIH and P-TEFb is an exciting possibility.

\section{Materials and methods}

Cloning of the small subunit of Drosophila P-TEFb

Drosophila P-TEFb was purified as described (Marshall and Price 1995). The small subunit was excised after SDS-PAGE and subjected to peptide sequencing (Keck Facility, Yale U niversity, $\mathrm{N}$ ew Haven, CT). Based on peptide sequences, two degenerate PCR primers (GGAATTCNATGYTNCARCARCC and AACTGCAGTCCARAARAARTCRTGRTT) were synthesized and used to amplify cDNA from Drosophila $K_{c}$ cell total $C D N A$ made from poly(A $)^{+}$RN A. A 1.1-kb cDN A fragment was amplified by PCR using Taq DNA polymerase. After digestion with Pstl (an internal site in the PCR product and a site designed in the $3^{\prime}$ end), the $0.7-k b$ fragment from the $3^{\prime}$ portion of the PCR product was cloned into the Bluescript II SK + vector (Stratagene). The 3' region of the full-length cDNA sequence was obtained using a GIBCO $3^{\prime}$ RACE kit with two specific primers from the 0.7-kb fragment (5'-TGTCAAGGATCAAACCGGCTGTGAT and 5'-CGAATTCCAAGAAACGCATCGATGC). The $5^{\prime}$ region of the full-length CDNA was obtained using a GIBCO 5' RACE kit with three gene-specific primers from the 0.7-kb fragment (5'-AGACCTGCCAAATCGTGT, 5'-AGAAGGTGGATCTGTAACCATTCGT, and 5'-GGAATTCAGATCTCGATCAGATTCA). The entire 1.2-kb coding region of the small subunit was cloned using a GIBCO reverse transcription PCR kit. First, the CDNA encoding the small subunit was generated in a reverse transcription with a designed primer (5'-TTACTACTCGAGCTACCAAACCCGGTC) and purified template (Drosophila embryonic mRNA). Second, the coding sequence was produced in a PCR reaction using Vent DNA polymerase, two primers (5'-TAAGCAAGCTTCTATGGCGCACATGTCC and 5'-TTACTACTCGAGCTACCAAACCCGGTC), and the CDNA as the template. Third, the coding sequence was digested with HindIII and Xhol and cloned into pET-2la vector.

\section{Generation of antibodies to PITALRE}

PITALRE-CT antibodies were affinity-purified rabbit IgG directed to the carboxy-terminal 20 amino acids of PITALRE (Santa Cruz Biotechnology). Antibodies against the whole PITALRE were generated using purified recombinant protein as antigen. First, two primers (5'-GCAGGATCCAGAATTCCATATGGCAAAGCAGTACGACTCGG and 5'- pGATCTCGAGCCCTCAGAAGACGCGCTCAAA) were used in a PCR reaction to amplify the coding region of the CDNA of PITALRE. The human brain CDNA mix (Clontech Co.) was used as template. The PCR product was cloned into a pET2la vector ( $\mathrm{N}$ ovagen Co.). The final vector was amplified and transformed into DE3 (BL21)-competent cells for expression of PITALRE. The transformed DE3 cells were grown to an $\mathrm{OD}_{600}$ of 0.6 and induced with $1 \mathrm{~mm}$ IPTG. After $3 \mathrm{hr}$ induction, the cells were collected 
and lysed by passing through a French press three times. The lysate was subjected to centrifugation at $15,000 \mathrm{~g}$ for $30 \mathrm{~min}$. The pellet was solubilized in $20 \mathrm{~mm}$ Tris $(\mathrm{pH} 7.5), 0.1 \mathrm{M} \mathrm{N} \mathrm{aCl}$, and $7 \mathrm{M}$ urea and loaded onto a Mono Q column (Pharmacia Co.). The flowthrough fraction of the Mono $Q$ column was loaded onto a M ono S column (Pharmacia Co.); the flowthrough fraction of the Mono S column, in turn, was subjected to dialysis against PBS. The dialyzed solution was centrifuged at $15,000 \mathrm{~g}$ for $30 \mathrm{~min}$. The pellet was suspended in the same buffer and sent for generation of antibodies in rabbit (Pocono Rabbit Farm \& Laboratory, Inc.).

\section{Immunodepletion of human P-TEFb}

Immunodepletion was performed by passing protein A-Sepharose-precleared HNE (in $20 \mathrm{~mm}$ HEPES at pH 7.6, 15\% glycerol, $165 \mathrm{~mm} \mathrm{KCl}, 0.1 \mathrm{~mm}$ EDTA, $1 \mathrm{~mm}$ DTT, $0.1 \mathrm{~mm}$ PM SF) through two affinity col umns made with protein A-Sepharose beads prebound with anti-PITALRE-CT antibodies or control IgGs (affinity-purified rabbit anti-goat IgG; Sigma). For every $50 \mu$ l of HNE, $10 \mu \mathrm{l}$ of protein A beads containing $1 \mu \mathrm{g}$ of bound IgG was used. After depletion of $\mathrm{HNE}$, the antibody-containing beads were washed extensively with 100 times the bead volume of $20 \mathrm{~mm}$ HEPES (pH 7.6), 0.5\% N P-40, 1\% Triton X-100, 5 mm DTT, and $600 \mathrm{~mm}$ or indicated concentration of $\mathrm{N} \mathrm{aCl}$ and then washed with 25 times the bead volume of $20 \mathrm{~mm}$ HEPES (pH 7.6) and 1 mM DTT. The amounts of the washed beads used for the kinase assays and silver staining were the equivalent of 1 and $10 \mu \mathrm{l}$ of $H N E$, respectively.

\section{CTD kinase assay}

CTD kinase assays were performed in a $20-\mu \mathrm{l}$ reaction containing $20 \mathrm{~mm}$ HEPES (pH 7.6), $10 \mu \mathrm{M} \mathrm{ATP,} 5 \mu \mathrm{Ci}\left[\gamma^{3}{ }^{32} \mathrm{P}\right] \mathrm{ATP}, \sim 10 \mathrm{ng}$ of Drosophila RNA polymerase II, $5 \mathrm{~mm} \mathrm{MgCl}_{2}$, and purified Drosophila P-TEFb or immunoprecipitated human P-TEFb. In the reactions containing DRB it was added to $50 \mu \mathrm{m}$ unl ess other indicated amounts were used. The reactions were incubated at $30^{\circ} \mathrm{C}$ for $1 \mathrm{hr}$.

\section{TAK activity assay}

Preparation of Tat fusion proteins and the TAK pull-down assay were conducted as described by Herrmann and Rice $(1993,1995)$ with modifications. Nuclear extract, cytoplasmic extract, or fractions containing partially purified TAK were incubated with glutathione-Sepharose beads containing GST-Tat fusion proteins for $1 \mathrm{hr}$ at $4^{\circ} \mathrm{C}$ with gentle rocking. DE3 (BL21) bacteria containing the GST-T at expression vectors were obtained from NIH AIDS Research and Reference Reagent Program. For maximum sensitivity, GST-Tat48 $\Delta$ was used unless specified otherwise. The beads ( $30 \mu \mathrm{l}, 50 \%$ slurry) were washed six to eight times with $1 \mathrm{ml}$ of EBCD buffer (50 mM Tris at pH 8.0, $120 \mathrm{~mm}$ $\mathrm{NaCl}, 0.5 \% \mathrm{NP}-40,5 \mathrm{~mm}$ DTT) containing $0.03 \% \mathrm{SDS}$, then two to four times with Tat kinase buffer (TKB/M g: 50 mM Tris$\mathrm{HCl}$ at $\mathrm{pH} 7.6,5 \mathrm{~mm}$ DTT, $5 \mathrm{~mm} \mathrm{MnCl}_{2}$, and $4 \mathrm{~mm} \mathrm{MgCl}_{2}$ ) and brought to $50 \mu \mathrm{l}$ with $\mathrm{TKB} / \mathrm{Mg}$ buffer and kinase assay mix. The final reaction contained $2 \mu$ M ATP, $10 \mu \mathrm{Ci}\left[\gamma^{-32}{ }^{32}\right] A T P$ (ICN, $3000 \mathrm{Ci} / \mathrm{mmole}$ ), and 50-100 $\mu \mathrm{M}$ CTD trimer peptide CTD 3 (ACSY SPT SPSY SPT SPSY SPT SPSKK). Reactions were incubated at $25^{\circ} \mathrm{C}$ for $40 \mathrm{~min}$, stopped by boiling in Laemmli sample buffer, and resolved by electrophoresis in $15 \%$ polyacrylamide/ bis-acrylamide (30\%:0.15\%) gels.

\section{Partial purification of TAK}

TAK was partially purified from a HeLa cell cytoplasmic fraction (2.6 grams of protein) prepared according to Ausubel et al. (1989), except that dialysis was omitted. Proteins precipitating between $10 \%$ and $40 \%$ saturation of ammonium sulfate (816 mg) were resuspended in DEAE buffer ( $25 \mathrm{~mm}$ HEPES at pH 7.6, $150 \mathrm{~mm} \mathrm{KCl}, 0.1 \mathrm{~mm}$ EDTA, $1 \mathrm{~mm}$ DTT, $0.1 \mathrm{~mm}$ PM SF, $4 \mathrm{~mm}$ $\mathrm{MgCl}_{2}, 1 \mu \mathrm{g} / \mathrm{ml}$ each of aprotinin, leupeptin, pepstatin A, and $10 \%$ glycerol), dialyzed against the same buffer, and applied to a 230-ml DEAE-Sepharose column equilibrated in DEAE buffer. The flowthrough fraction (350 mg of protein) was concentrated by $50 \%$ ammonium sulfate precipitation. The proteins were resuspended in HE buffer (same as DEAE buffer, except $25 \mathrm{~mm}$ HEPES at pH 6.9, and $100 \mathrm{~mm} \mathrm{KCl}$ were used) and loaded onto a perfusion chromatography heparin affinity column (POROS $20 \mathrm{HE}$; PerSeptive Biosystems). The column was washed with the same buffer, and proteins were el uted with a linear gradient of $100-500 \mathrm{~mm} \mathrm{KCl}$ in 15 column volumes of HE buffer. TAK activity eluted from 200 to $250 \mathrm{mM} \mathrm{KCl}$. Active fractions were diluted with an equal volume of the same buffer, except that the $\mathrm{pH}$ was 8.0 and $\mathrm{KCl}$ was omitted, and loaded onto a perfusion chromatography cation exchange column (POROS 20 SP; PerSeptive Biosystems). The column was washed with SP buffer (the same as HE buffer, except that the buffer was $25 \mathrm{~mm}$ HEPES at $\mathrm{pH} 7.5$ ) and el uted with a linear gradient of $100-500 \mathrm{mM} \mathrm{KCl}$ in 15 column volumes of SP buffer. The active fractions ( $1 \mathrm{mg}$ of protein) eluted at $\sim 300 \mathrm{~mm} \mathrm{KCl}$.

\section{Transcription assay}

The transcription template ( $\mathrm{Ncol}$-digested pLTR-4/CAT vector containing the HIV-1 LTR from -153 to +80 , provided by $P$. Luciw, University of California, Davis) was used in a pulsechase transcription experiment (Fig. 2). Reactions [12 $\mu \mathrm{l}$ total; $20 \mathrm{~mm}$ HEPES at pH 7.6, $7 \mathrm{~mm} \mathrm{M} \mathrm{gCl}, 20 \mu \mathrm{g} / \mathrm{ml}$ of DNA template, $3 \mu \mathrm{l}$ of $\mathrm{HNE}, 64 \mathrm{~mm} \mathrm{KCl}$, with $50 \mu \mathrm{M}$ DRB or $0.3 \mu \mathrm{l}$ of Drosophila P-TEFb (Marshall et al. 1996), as indicated] were preincubated for $20 \mathrm{~min}$ at $30^{\circ} \mathrm{C}$, pulsed for $2 \mathrm{~min}$ by adding ATP, GTP, and UTP to $600 \mu \mathrm{M}$ each and $5 \mu \mathrm{Ci}\left[\alpha-{ }^{32}\right.$ P]CTP $(\sim 0.1$ $\mu \mathrm{M})$, and chased for $5 \mathrm{~min}$ by adding CTP to $1.2 \mathrm{~mm}$. Reactions were stopped, phenol extracted, and analyzed on a $6 \%$ polyacrylamide gel as described ( $M$ arshall and Price 1995). pLTRTAR-Luc and pLTR- $\Delta$ TAR-Luc plasmids were digested with EcoRI to generate TAR and $\triangle T A R$ template that were used in the experiments shown in Figure 7. The TAR template contains HIV-1 LTR from -475 to +76 , whereas the $\Delta$ TAR template contains HIV-1 LTR from -475 to +19 . Transcription with TAR and $\triangle T A R$ templates will generate runoffs of 694 and 646 nucleotides, respectively. In Figure 7A, the transcription reaction mixture contained $20 \mathrm{~mm}$ HEPES (pH 7.6), $7 \mathrm{~mm} \mathrm{M} \mathrm{gCl} 2,60 \mathrm{~mm} \mathrm{KCl}$, $0.5 \mu \mathrm{l}$ of HNE (or depHNE as indicated), $20 \mu \mathrm{g} / \mathrm{ml}$ of TAR template (or $\triangle T A R$ template as indicated), an indicated amount of HIV-1 Tat (86-residue HIV-1 Tat followed by a streptavidin binding tag at its carboxyl terminus, kindly provided by $O$. Flores, Tularik, Inc., South San Francisco, CA), and DRB. The reaction mix was preincubated for $15 \mathrm{~min}$ at $30^{\circ} \mathrm{C}$, and the transcription was started by adding nucl eotides to final concentrations of $50 \mu \mathrm{M}$ ATP, $50 \mu \mathrm{M}$ GTP, $50 \mu \mathrm{M}$ UTP, $10 \mu \mathrm{M}$ CTP, and $5 \mu \mathrm{Ci}\left[\alpha{ }^{32} \mathrm{P}\right] \mathrm{CTP}(\sim 0.1 \mu \mathrm{M})$ and continuously labeling for $20 \mathrm{~min}$ (Fig. 7A), or by adding nucleotides to final concentrations of 50 $\mu \mathrm{MM}$ ATP, $50 \mu \mathrm{M}$ GTP, $50 \mu \mathrm{M}$ UTP, and $5 \mu \mathrm{Ci}\left[\alpha^{-32} \mathrm{P}\right] \mathrm{CTP}(\sim 0.1$ $\mu \mathrm{M}$ ), and pulsed for 2 min (Fig. 7B). The reactions were stopped, and the reaction mixtures were phenol-extracted. The transcripts were then analyzed on a $6 \%$ polyacrylamide gel as described (Marshall and Price 1995). 


\section{Acknowledgments}

We thank Ken Williams at the Keck Foundation Biotechnology Resource Laboratory at Yale University for providing an excellent protein sequencing service, Osvaldo Flores for providing Tat protein, and Ofer Gileadi for providing the CTD3 peptide. We also thank Izhak Haviv and Yosef Shaul for fruitful discussions and help with purification, and Pam Geyer for critical reading of the manuscript. This work was supported by $\mathrm{Na}$ tional Institutes of Health grants GM35500, A131802, and TW00506.

The publication costs of this article were defrayed in part by payment of page charges. This article must therefore be hereby marked "advertisement" in accordance with 18 USC section 1734 solely to indicate this fact.

\section{References}

Aso, T., W.S. Lane, J.W. Conaway, and R.C. Conaway. 1995. Elongin (SIII): A multisubunit regulator of elongation by RN A polymerase II. Science 269: 1439-1443.

Ausubel, F.M., R. Brent, R.E. Kingston, D.D. Moore, J.G. Seidman, J.A. Smith, and K. Struhl. 1989. Current protocols in molecular biology Greene Publishing Associates/WileyIntersience, N ew York, NY.

Bentley, D.L. 1995. Regulation of transcriptional el ongation by RNA polymerase II. Curr. Opin. Genet. Dev. 5: 210-216.

Blau, J., H. Xiao, S. M cCracken, P. O'Hare, J. Greenblatt, and D. Bentley. 1996. Three functional classes of transcriptional activation domains. Mol. Cell. Biol. 16: 2044-2055.

Braddock, M., A.M. Thorburn, A.J. Kingsman, and S.M. Kingsman. 1991. Blocking of Tat-dependent HIV-1 RNA modification by an inhibitor of RNA polymerase II processivity. Nature 350: 439-441.

Carroll, R., B.M. Peterlin, and D. Derse. 1992. Inhibition of human immunodeficiency virus type 1 Tat activity by coexpression of heterologous trans activators. J. Virol. 66: 20002007.

Chun, R.F. and K.T. Jeang. 1996. Requirements for RN A polymerase II carboxyl-terminal domain for transcription of human retroviruses, human $\mathrm{T}$-cell Iymphotrophic virus, and HIV-1. J. Biol. Chem. 271: 27888-27894.

Cujec, T.P., H. Cho, E. Maldonado, J. Meyer, D. Reinberg, and B.M. Peterlin. 1997. The human immunodeficiency virus transactivator Tat interacts with the RN A polymerase II hoIoenzyme. Mol. Cell. Biol. 17: 1817-1823.

Cullen, B.R. 1993. Does HIV-1 Tat induce a change in viral initiation rights? Cell 73: 417-420.

Dahmus, M.E. 1996. Reversible phosphorylation of the c-terminal domain of RNA polymerase II. J. Biol. Chem. 271: 19009-19012.

Egyhazi, E., A. Ossoinak, A. Pigon, C. Holmgren, J.M. Lee, and A.L. Greenleaf. 1996. Phosphorylation dependence of the initiation of productive transcription of Bal biani ring 2 genes in living cells. Chromosoma 104: 422-433.

Feinberg, M.B., D. Baltimore, and A.D. Frankel. 1991. The role of Tat in the human immunodeficiency virus life cycle indicates a primary effect on transcriptional el ongation. Proc. Natl. Acad. Sci. 88: 4045-4049.

Garcia-Martinez, L.F., D. Ivanov, and R.B. Gaynor. 1997a. Association of Tat with purified HIV-1 and HIV-2 transcription preinitiation complexes. J. Biol. Chem. 272: 6951-6958.

Garcia-M artinez, L.F., G. M avankal, J.M. N eveu, W.S. Lane, D. Ivanov, and R.B. Gaynor. 1997b. Purification of a Tat-associated kinase reveals a TFIIH complex that modulates HIV-1 transcription. EMBO J. 16: 2836-2850.
Garriga, J., X. Mayol, and X. Grana. 1996a. The CDC2-related kinase PITALRE is the catalytic subunit of active multimeric protein complexes. Biochem. J. 319: 293-298.

Garriga, J., E. Segura, X. Mayol, C. Grubmeyer, and X. Grana. 1996b. Phosphorylation site specificity of the CDC2-related kinase PITALRE. Biochem. J. 320: 983-989.

Goodrich, J.A. and R. Tjian. 1994. Transcription factors IIE and $\mathrm{IIH}$ and ATP hydrolysis direct promoter clearance by RNA polymerase II. Cell 77: 145-156.

Grana, X., A. De Luca, N. Sang, Y. Fu, P.P. Claudio, J. Rosenblatt, D.O. Morgan, and A. Giordano. 1994. PITALRE, a nuclear CDC2-related protein kinase that phosphorylates the retinoblastoma protein in vitro. Proc. Natl. Acad. Sci. 91: 3834-3838.

Guo, H. and D.H. Price. 1993. Mechanism of DmS-II-mediated pause suppression by Drosophila RN A polymerase II. J. Biol. Chem. 268: 18762-18770.

Herrmann, C.H. and A.P. Rice. 1993. Specific interaction of the human immunodeficiency virus Tat proteins with a cellular protein kinase. Virology 197: 601-608.

- - - 1995. Lentivirus Tat proteins specifically associate with a cellular protein kinase, TAK, that hyperphosphorylates the carboxyl-terminal domain of the large subunit of RN A polymerase II: Candidate for a Tat cofactor. J. Virol. 69: 16121620.

Irie, K., S. N omoto, I. M iyajima, and K. M atsumoto. 1991. SGV1 encodes a CDC28/ cdc2-related kinase required for a $\mathrm{G}$ al pha subunit-mediated adaptive response to pheromone in $\mathrm{S}$. cerevisiae. Cell 65: 785-795.

Jeang, K.-T., R. Chun, N.H. Lin, A. Gatignol, C.G. Glabe, and H. Fan. 1993. In vitro and in vivo binding of human immunodeficiency virus type 1 Tat protein and Sp1 transcription factor. J. Virol. 67: 6224-6233.

Jones, K.A. and B.M. Peterlin. 1994. Control of RN A initiation and el ongation at the HIV-1 promoter. Annu. Rev. Biochem. 63: 717-743.

Kao, S.-Y., A.F. Calman, P.A. Luciw, and E.M. Peterlin. 1987. Antitermination of transcription within the long terminal repeat of HIV by the tat gene product. Nature 330: 489-493.

Kashanchi, F., G. Piras, M.F. Radonovich, J.F. Duvall, A. Fattaey, C.M. Chiang, R.G. Roeder, and J.N. Brady. 1994. Direct interaction of human TFIID with the HIV-1 transactivator tat. Nature 367: 295-299.

Keen, N.J., M.J. Gait, and J. Karn. 1996. Human immunodeficiency virus type-1 Tat is an integral component of the activated transcription-elongation complex. Proc. Natl. Acad. Sci. 93: 2505-2510.

Kephart, D.D., B.Q. Wang, Z.F. Burton, and D.H. Price. 1994. Functional analysis of Drosophila factor 5 (TFIIF), a general transcription factor. J. Biol. Chem. 269: 13536-13543.

Kerppola, T.K. and C.M. Kane. 1991. RN A polymerase: Regulation of transcript elongation and termination. FASEB J. 5: 2833-2842.

Laspia, M.F., A.P. Rice, and M.B. Mathews. 1989. HIV-1 Tat protein increases transcriptional initiation and stabilizes elongation. Cell 59: 283-292.

Laspia, M.F., A.P. Rice, and M.B. Mathews. 1990. Synergy between HIV-1 Tat and adenovirus E1A is principally due to stabilization of transcriptional elongation. Genes \& Dev. 4: 2397-2408.

Laspia, M.F., P. Wendel, and M.B. Mathews. 1993. HIV-1 Tat overcomes inefficient transcriptional el ongation in vitro. J. Mol. Biol. 232: 732-746.

Lee, J.M. and A.L. Greenleaf. 1997. Modulation of RNA polymerase II elongation efficiency by c-terminal heptapeptide repeat domain kinase I. J. Biol. Chem. 272: 10990-10993. 
Madore, S.J. and B.R. Cullen. 1993. Genetic analysis of the cofactor requirement for human immunodeficiency virus type 1 Tat function. J. Virol. 67: 3703-3711.

Marciniak, R.A. and P.A. Sharp. 1991. HIV-1 Tat protein promotes formation of more-processive el ongation complexes. EMBO J. 10: 4189-4196.

Marshall, N.F. and D.H. Price. 1992. Control of formation of two distinct classes of RNA polymerase II elongation complexes. Mol. Cell. Biol. 12: 2078-2090.

- - . 1995. Purification of P-TEFb, a transcription factor re quired for the transition into productive elongation. J. Biol. Chem. 270: 12335-12338.

Marshall, N .F., J.M. Peng, Z. Xie, and D.H. Price. 1996. Control of RNA polymerase II elongation potential by a novel carboxyl-terminal domain kinase. J. Biol. Chem. 271: 2717627183.

Mavankal, G., S.H.I. Ou, H. Oliver, D. Sigman, and R.B. Gaynor. 1996. Transcription factors, and gene expression. Human immunodeficiency virus type 1 and 2 Tat proteins specifically interact with RN A polymerase II. Proc. Natl. Acad. Sci. 93: 2089-2094.

O'Brien, T., S. Hardin, A. Greenleaf, and J.T. Lis. 1994. Phosphorylation of RNA polymerase II C-terminal domain and transcriptional el ongation. N ature 370: 75-77.

Parada, C.A. and R.G. Roeder. 1996. Enhanced processivity of RN A polymerase II triggered by Tat-induced phosphorylation of its carboxy-terminal domain. Nature 384: 375-378.

Pendergrast, P.S. and N. Hernandez. 1997. RN A-targeted activators, but not DNA-targeted activators, repress the synthesis of short transcripts at the human immunodeficiency virus type 1 long terminal repeat. J. Virol. 71: 910-917.

Price, D.H., A.E. Sluder, and A.L. Greenleaf. 1987. Fractionation of transcription factors for RNA polymerase II from Drosophila $K_{c}$ cell nuclear extracts. J. Biol. Chem. 262: 32443255.

Reines, D., J.W. Conaway, and R.C. Conaway. 1996. The RN A polymerase II general elongation factors. Trends Biochem. Sci. 21: 351-355.

Rice, A.P. and F. Carlotti. 1990. Structural analysis of wild-type and mutant human immunodeficiency virus type 1 Tat proteins. J. Virol. 64: 6018-6026.

Shel don, M., R. Ratnasabapathy, and N. Hernandez. 1993. Characterization of the inducer of short transcripts, a human immunodeficiency virus type 1 transcriptional element that activates the synthesis of short RNAs. Mol. Cell. Biol. 13: 1251-1263.

Shilatifard, A., W.S. Lane, K.W. Jackson, R.C. Conaway, and J.W. Conaway. 1996. An RNA polymerase II elongation factor encoded by the human ELL gene. Science 271: 18731876.

Spencer, C.A. and M. Groudine. 1990. Transcription el ongation and eukaryotic gene regulation. Oncogene 5: 777-786.

Sterner, D.E., J.M. Lee, S.E. Hardin, and A.L. Greenleaf. 1995. The yeast carboxyl-terminal repeat domain kinase CTDK-I is a divergent cyclin-cyclin-dependent kinase complex. Mol. Cell. Biol. 15: 5716-5724.

Tamm, I. and T. Kikuchi. 1979. Early termination of heterogeneous nuclear RN A transcripts in mammalian cells: accentuation by 5,6-dichloro 1-beta-D-ribofuranosylbenzimidazole. Proc. Natl. Acad. Sci. 76: 5750-5754.

Tsuchiya, H., T. Iseda, and O. Hino. 1996. Identification of a novel protein (VBP-1) binding to the vonHippel-Lindau (VHL) tumor suppressor gene product. Cancer Res. 56: 28812885.

Veschambre, P., P. Simard, and P. Jalinot. 1995. Evidence for functional interaction between the HIV-1 T at transactivator and the TATA box binding protein in vivo. J. Mol. Biol. 250: 169-180.

Wright, S. 1993. Regulation of eukaryotic gene expression by transcriptional attenuation. Mol. Biol. Cell 4: 661-668.

Xie, Z. and D.H. Price. 1996. Purification of an RN A polymerase II transcript release factor from Drosophila. J. Biol. Chem. 271: 11043-11046.

Yang, X.Z., C.H. Herrmann, and A.P. Rice. 1996. The human immunodeficiency virus tat proteins specifically associate with TAK in vivo and require the carboxy-terminal domain of RN A polymerase II for function. J. Virol. 70: 4576-4584.

Yankulov, K., K. Yamashita, R. Roy, J.M. Egly, and D.L. Bentley. 1995. The transcriptional el ongation inhibitor 5,6-dichloro$1-\beta-D$-ribofuranosylbenzimidazole inhibits transcription factor IIH-associated protein kinase. J. Biol. Chem. 270: 23922-23925.

Yankulov, K.Y., M. Pandes, S. McCracken, D. Bouchard, and D.L. Bentley. 1996. TFIIH functions in regulating transcriptional el ongation by RN A polymerase II in Xenopus oocytes. Mol. Cell. Biol. 16: 3291-3299.

Zhou, Q.A. and P.A. Sharp. 1996. TAT-SF1 - cofactor for stimuIation of transcriptional elongation by HIV-1 Tat. Science 274: 605-610. 


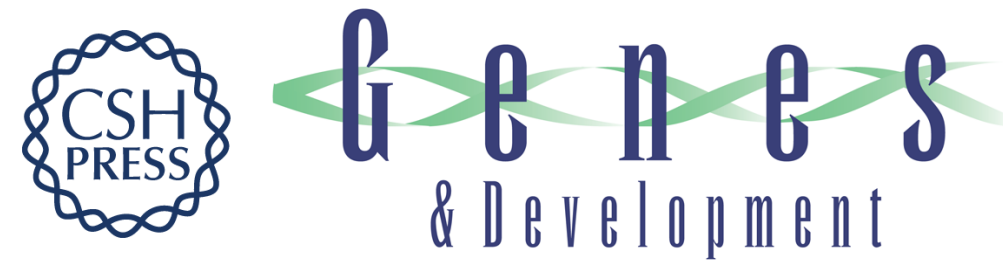

\section{Transcription elongation factor $\mathrm{P}-\mathrm{TEFb}$ is required for $\mathrm{HIV-1}$ Tat transactivation in vitro}

Yuerong Zhu, Tsafrira Pe'ery, Junmin Peng, et al.

Genes Dev. 1997, 11:

Access the most recent version at doi:10.1101/gad.11.20.2622

References

This article cites 55 articles, 36 of which can be accessed free at: http://genesdev.cshlp.org/content/11/20/2622.full.html\#ref-list-1

License

Email Alerting

Receive free email alerts when new articles cite this article - sign up in the box at the top Service right corner of the article or click here.

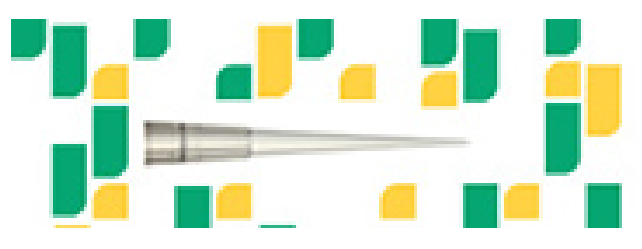

Focused on your science. 